\title{
miR-185-5p inhibits F-actin polymerization and reverses epithelial mesenchymal transition of human breast cancer cells by modulating RAGE
}

\author{
CHONGGAO YIN ${ }^{1,2}$, GUOXIN ZHANG $^{3}$, RUIMEI SUN $^{3}$, XINTING PAN $^{4}$, \\ XUEWEN WANG ${ }^{3}$, HONGLI LI ${ }^{3}$ and YUNBO SUN ${ }^{4}$ \\ ${ }^{1}$ College of Nursing, Qingdao University, Qingdao, Shandong 266003; ${ }^{2}$ College of Nursing; ${ }^{3}$ Medicine Research Center, \\ Weifang Medical University, Weifang, Shandong 261053; ${ }^{4}$ CU, Affiliated Hospital of Qingdao University, \\ Qingdao, Shandong 266003, P.R. China
}

Received December 16, 2017; Accepted May 10, 2018

DOI: $10.3892 / \mathrm{mmr} .2018 .9294$

\begin{abstract}
In our previous study, advanced glycosylation end-product specific receptor (RAGE) was observed to bind to S100A8/A9 and cause epithelial mesenchymal transition (EMT). The results from target gene prediction revealed that microRNA (miR)-185-5p had a RAGE binding site. However, the function of miR-185-5p in the invasion and migration of breast cancer remains ambiguous. In the present study, the expression of miR-185-5p was examined in breast cancer tissues and cells. Clinical features revealed a negative correlation between miR-185-5p and tumor size, as well as in tumor differentiation and lymph node metastasis in breast cancer. In addition, miR-185-5p was negatively associated with RAGE, and this miRNA reversed the EMT of breast cancer by modulating RAGE in vitro. In addition, miR-185-5p inhibited the S100A8/A9-induced EMT of breast cancer cells by the nuclear factor- $\mathrm{kB} /$ Snail signaling pathway. Notably, miR-185-5p upregulation inhibited the F-actin polymerization induced by S100A8/A9 in breast cancer. Furthermore, overexpression of miR-185-5p and reduction of RAGE inhibited lung metastasis node in vivo. Thus, miR-185-5p represents a potential therapeutic target in breast cancer by modulating RAGE.
\end{abstract}

Correspondence to: Professor Yunbo Sun, ICU, Affiliated Hospital of Qingdao University, 16 Road Jiang Su, Qingdao, Shandong 266003, P.R. China

E-mail: sunyunbo163@163.com

Professor Hongli Li, Medicine Research Center, Weifang Medical University, 7166 Baotong Xi Street, Weifang, Shandong 261053, P.R. China

E-mail: ycglihongli@163.com

Key words: microRNA-185-5p, advanced glycosylation end-product specific receptor, epithelial mesenchymal transition, F-actin polymerization, S100A8/A9

\section{Introduction}

Breast cancer is one of the most frequently diagnosed cancers and the leading contributing factor in cancer death among females (1). In spite of significantly improved diagnosis and clinical treatment strategies, low survival and high recurrence rate persist in many breast cancer patients. Invasion and metastasis are the primary reasons for mortality caused by breast cancer. However, the precise molecular mechanism of breast cancer invasion and metastasis remains ambiguous.

The receptor for advanced glycation end products (RAGE), which is a member of the immune globulin family, was isolated from the bovine lung endothelium (2). RAGE is a multiple ligand and pattern recognition receptor that has been implicated in the progression of various human cancers because of its ability to drive tumor growth and progression (3). Meanwhile, many clinical studies have indicated that higher RAGE is related to various malignant tumors, such as prostate cancer and glioma $(4,5)$. RAGE has been implicated as a potential mechanism driving the development, progression, and metastasis of breast cancer (6-8).

Epithelial mesenchymal transition (EMT) is indispensable to increasing invasion and migration during the development of a malignant tumor. EMT can be affected by various transcription factors and is accompanied by changes in the EMT marker protein. In EMT, cancer cells exhibit the property of mesenchymal cells; that is, the cells diffuse from the primary tumor and invade adjacent tissues and blood vessels $(9,10)$. Recent studies have demonstrated that microRNA (miRNA)s are key factors in EMT-associated cancer invasion and metastasis.

miRNAs belong to a family of small non-coding RNAs with approximately 22-25 nucleotides in length. Functional studies have demonstrated that miRNA serves as a modulating gene that can regulate target mRNA to repress translation. Several miRNAs, such as miR-200c (11), miR-34 (12), and miR-9 (13), enhance or repress metastasis and invasion by regulating EMT. miR-185-5p has been reported to be downregulated in NSCLC (14), gastric cancer (GC) (15), breast cancer (16), and glioma (17). Plasma miR-185 is decreased in patients with 
esophageal squamous cell carcinoma and might suppress tumor migration and invasion by targeting RAGE (18). However, the functional mechanism of miR-185-5p targeting the RAGE mRNA in breast cancer is unknown. Thus, understanding the potential mechanism of the invasion and EMT of breast cancer may be an effective therapeutic strategy.

In this study, we first identified a negative correlation between miR-185-5p and RAGE expressed in breast cancer tissues and cells. Upregulation or downregulation of miR-185-5p in vitro and in vivo resulted in the significant inhibition or promotion of invasion and EMT. Furthermore, upregulation of miR-185-5p impacted F-actin polymerization caused by S100A8/A9 of breast cancer cells. Finally, the results indicated that $\mathrm{miR}-185-5 \mathrm{p}$, as an anti-oncogene, played a pivotal role by targeting RAGE in breast cancer. Hence, miR-185-5p could act as a rationale for the diagnosis and therapy of breast cancer patients.

\section{Materials and methods}

Clinical samples. Fresh frozen breast tissue specimens and adjacent normal breast tissue samples were acquired from the Affiliated Hospital of the Weifang Medical University (Shandong, China). Patients did not undergo chemotherapy or radiotherapy before surgery. Written informed consent was acquired from all patients, and the study was approved by the Institute Research Ethics Committee at the Cancer Center, Weifang Medical University.

Cell culture. All human breast cancer cell lines, namely, MDA-MB-231 (MDA231), T47D, MDA-MB-453 (MDA453), SK-BR-3 and MCF-7, and human normal breast epithelial cell MCF-10A were acquired from ATCC (USA). The MCF-10A cells were cultured in DMEM/F12, which contained $10 \% \mathrm{FBS}$, $20 \mathrm{ng} / \mathrm{ml} \mathrm{EGF}, 0.1 \mathrm{mg} / \mathrm{ml} \mathrm{CT}, 10 \mathrm{mg} / \mathrm{ml}$ insulin, and $500 \mathrm{ng} / \mathrm{ml}$ hydrocortisone. MCF-7 cells were cultured in MEM with $10 \%$ FBS and $1 \%$ sodium pyruvate. T47D and SK-BR-3 cells were cultured in DMEM with $10 \%$ FBS. MDA231 cells were cultured in RPMI-1640. MDA453 cells were cultured in L-15 with $10 \% \mathrm{FBS}$. All cell cultures were incubated at $37^{\circ} \mathrm{C}$ under $5 \% \mathrm{CO}_{2}$ atmosphere.

Plasmid construction and cell transfection. MDA231 cells or MCF-7 cells $\left(2 \times 10^{5}\right)$ were planted in six-well plates overnight to ensure that cell confluence could reach $60-80 \%$ at the time of transfection. Lipofectamine 2000 (Invitrogen; Thermo Fisher Scientific Inc., Waltham, MA, USA) was employed in cell transfection according to the manufacturer's protocol. miR-185-5p mimics, negative control mimics (NCs), miR-185-5p inhibitor, and anti-NC were synthesized by Genechem, Inc. (Daejeon, Korea). The siRNA sequence of RAGE and a control vector were synthesized by Genechem Inc. (8). The transfected cells were selected for 14 days using $600 \mu \mathrm{g} / \mathrm{ml} \mathrm{G} 418$. We isolated single cell culture and maintained the stable transfected cells using $300 \mu \mathrm{g} / \mathrm{ml} \mathrm{G} 418$. The stable transfected cells were used for subsequent studies.

Western blot analysis. The cells or tissues were lysed using RIPA buffer with protein phosphatase inhibitors for western blot. Whole cell or tissue protein lysates were electrophoresed on $10 \%$ SDS-PAGE and transferred onto PVDF membranes (Millipore, Billerica, MA, USA). The membranes were subsequently incubated with primary antibodies overnight, washed thrice, incubated with secondary antibody, and exposed to ECL. We analyzed the samples using ImageJ software (NIH, Bethesda, Maryland). The relative protein levels were quantified using three different western blots. The following antibodies were employed: RAGE (1:1,000; cat. no. 6996), E-cadherin (1:1,000; cat. no. 3195), vimentin (1:1,000; cat. no. 5741; all Cell Signaling Technology, Inc. Cell Signaling Technology, Inc., Boston, MA, USA), Snail (1:500; cat. no. ab53519), Slug (1:500; cat. no. ab27568), twist-related protein (Twist; 1:500; cat. no. ab50581), zinc finger E-box binding homeobox (Zeb)1 (1:500; cat. no. ab124512; all Abcam, Cambridge, UK), Zeb2 (1:500; cat. no. sc48789; Santa Cruz Biotechnology, Inc., Dallas, TX, USA), HRP-conjugated anti-rabbit IgG, and anti-mouse IgG antibody (1:4,000; cat. nos. 7074 and 7076; Cell Signaling Technology, Inc.). The western blot results were from at least three repeated experiments.

Cell invasion. The Transwell chamber invasion assay was spread in the Matrigel. Breast cancer cells were mixed and incubated at $37^{\circ} \mathrm{C}$ for $24 \mathrm{~h}$. Then, the cells were washed thrice and fixed with $4 \%$ paraformaldehyde for $15 \mathrm{~min}$ and stained with Giemsa for $35 \mathrm{~min}$. Five view pictures were randomly selected, and the cells were counted under a light microscope (magnification, x200).

Reverse transcription-quantitative polymerase chain reaction (RT-qPCR). Total RNAs were isolated using TRIzol reagent. RT-qPCR was conducted using SYBR-Green on an Applied Biosystems 7500 (Applied Biosystems; Thermo Fisher Scientific, Inc.). Expression of U6 was used as the internal control. The relative mRNA expression was normalized using the $2^{-\Delta \Delta \mathrm{Cq}}$ method (19). The thermocycling conditions for RT-qPCR were as follows: $95^{\circ} \mathrm{C}$ for $20 \mathrm{sec}$, followed by 35 cycles of $95^{\circ} \mathrm{C}$ for $5 \mathrm{sec}, 63^{\circ} \mathrm{C}$ for $30 \mathrm{sec}$ and $72^{\circ} \mathrm{C}$ for 5 sec. The sequence of the RT primer was 5'-GTCGTATCC AGTGCAGGGTCCGAGGTATTCGCACTGGATACGACT CAGGA-3'. Specific primers for miR-185-5p were as follows: Forward, 5'-GCGCGATTGGAGAGAAAGGCAGT-3' and reverse, 5'-ATCCAGTGCAGGGTCCGAGG-3'.

Luciferase reporter assay. TargetScan 6.0 and miRNA. org were used to predict sequences of miR-185-5p and pair 3'-untranslated region (UTR) sequences of RAGE. MDA231 cells or MCF-7 cells $\left(3 \times 10^{4}\right)$ were planted in triplicates in 24-well plates for $24 \mathrm{~h}$ before transfection. Then, $100 \mathrm{ng}$ of pGL3-RAGE-3'UTR wild-type (WT) or pGL3-RAGE-3'UTR-mutant (MUT), plus $1 \mathrm{ng}$ of pRL-TK (Promega, Madison, WI, USA) and the matched miR-185-5p, NC, anti-NC, or anti-miR-185-5p plasmid, were co-transfected using Lipofectamine 2000 in accordance with the manufacturer's protocol. Firefly luciferase activity was measured after $24 \mathrm{~h}$ of transfection.

Chemotaxis assay. S100A8/A9 was added into the bottom chamber, and $2 \times 10^{5}$ cells were added into the upper chamber. The chambers were incubated at $37^{\circ} \mathrm{C}$ under $5 \% \mathrm{CO}_{2}$. 


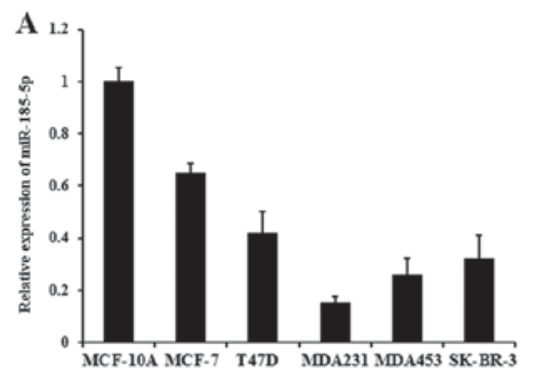

C

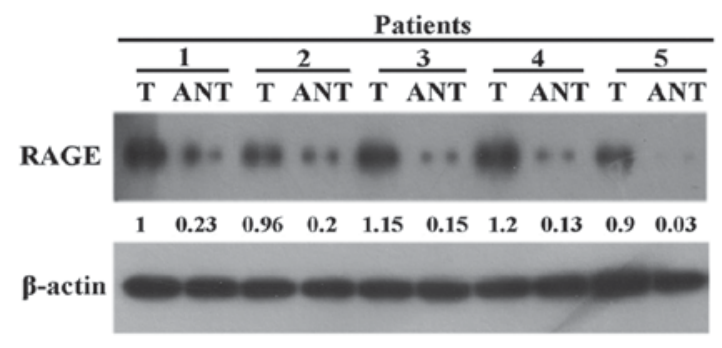

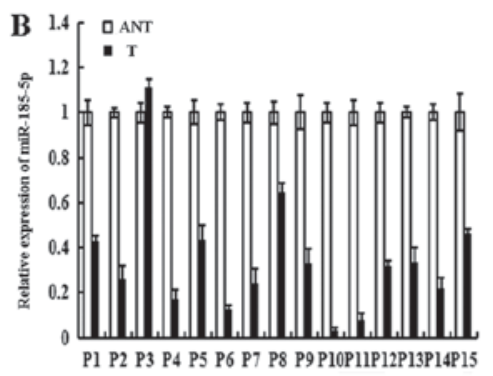
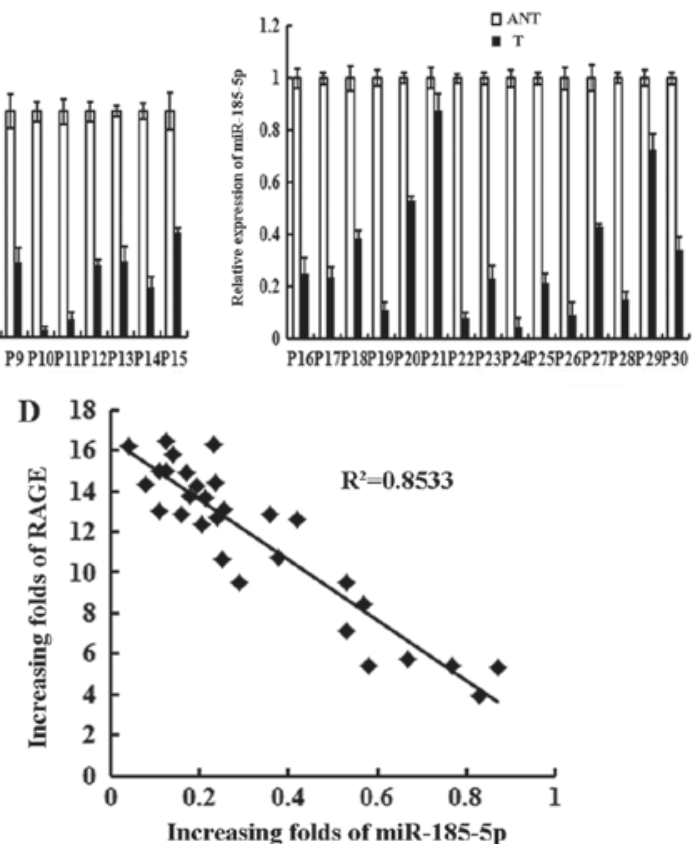

Figure 1. miR-185-5p is downregulated in metastatic patient tissues and cells and is correlated with the expression of RAGE. (A) The expression of miR-185-5p was detected in MCF-7, T47D, MDA231, MDA453, SK-BR-3 and MCF-10A cells. (B) Relative expression of miR-185-5p was tested in 30 pairs of fresh frozen tumors tissues (T group) and their adjacent normal tissues (ANT group). (C) Expression of RAGE compared between paired T tissues and ANTs tissues. The results of western blotting were from a representative of at least three repeated experiments. (D) Correlation of expression between miR-185-5p and RAGE. miR, microRNA; T, tumor tissues; ANT, adjacent normal tissues; P, patient number; RAGE, advanced glycosylation end-product specific receptor.

Subsequently, the polycarbonate filter membranes were fixed and stained using Giemsa. The numbers of migrating cells were calculated in five random fields using light microscopy, and the average number of cells was determined. This experiment was repeated thrice.

Cellular F-actin measurement. Breast cancer cells were immobilized with $4 \%$ paraformaldehyde and PBS. Then, the cells were washed thrice for $5 \mathrm{~min}$ at each instance. The cells were washed with PBS and blocked with buffer, which included goat serum, for $45 \mathrm{~min}$. The cells were stained with Rhodamine phalloidin for $1 \mathrm{~h}$, washed thrice, and covered with fluorescence decay resistant sealing tablets. F-actin content was measured with a fluorescence reader. The relative $\mathrm{F}$-actin content was calculated as follows: F-actin $\Delta \mathrm{t} / \mathrm{F}$-actin $0=$ fluorescence $\Delta t /$ fluorescence 0 .

Immunofluorescence. The cells were fixed for $20 \mathrm{~min}$ using $4 \%$ paraformaldehyde. Then, the cells were permeabilized with $0.1 \%$ Triton X-100 and blocked with $1 \%$ BSA for 45 min at $37^{\circ} \mathrm{C}$. The primary antibodies E-cadherin and vimentin were incubated overnight at $4^{\circ} \mathrm{C}$. Cy3 and FITC secondary antibodies and DAPI were used.

Animal studies. The Severe Combined Immune-deficiency (SCID) mice were approved by the Animal Care and Use Committee of Wei Fang Medical University. Female SCID mice (4-5 weeks old) were used. MDA231/NC, Scr/MDA231, MDA231/miR-185-5p, and SiRAGE/MDA231 cells were injected into the oxter of the SCID mice $(n=10)$. When the xenografts were evident, an intratumor injection of S100A8/A9 at $100 \mathrm{ng} / \mathrm{kg}$ was performed for 4 weeks. After
8 weeks, metastasis in the lung tissues was examined by H\&E staining.

Statistical analysis. Data were analyzed using SPSS v16.0 software (SPSS, Inc., Chicago, IL, USA). The results were presented as the mean \pm standard deviation. $\mathrm{X}^{2}$-test was used to analyze the associations between miR-185-5p and the clinicopathologic features. Student's t-test or analysis of variance with Dunnett's post hoc test were conducted to determine the statistical significance for comparisons between the groups. $\mathrm{P}<0.05$ was considered to indicate a statistically significant difference.

\section{Results}

Downregulation of miR-185-5p in metastatic patient tissues and cells was correlated with clinicopathological features of breast cancer. miR-185-5p is known to play a fatal role in various cancers, such as breast cancer. However, the biological role of miR-185-5p has not been fully elucidated. In the present study, we first analyzed the miR-185-5p expression in breast cancer cells by RT-qPCR. The results indicated that the expression of miR-185-5p was markedly downregulated in breast cancer cells compared with that in MCF-10A. The expression of miR-185-5p was higher in MCF-7 than in MDA231 (Fig. 1A). The expression of miR-185-5p in 30 selected fresh breast cancer tissues (T) and paired adjacent non-tumor tissues (ANTs) was tested using RT-qPCR to further investigate the clinical relevance of miR-185-5p. The results demonstrated that miR-185-5p was significantly reduced in all 30 tumor tissues compared with the paired ANTs (Fig. 1B). Consequently, RAGE was markedly increased in most of the fresh tumor tissues relative to the paired ANTs (Fig. 1C). Thus, the expression of miR-185-5p 
Table I. Associations between clinical features and miRNA-185-5p expression in breast cancer patients.

\begin{tabular}{|c|c|c|c|}
\hline \multirow[b]{2}{*}{ Variables } & \multicolumn{2}{|c|}{ miR-185-5p } & \multirow[b]{2}{*}{ P-value } \\
\hline & High expression (n) & Low expression (n) & \\
\hline \multicolumn{4}{|l|}{ Age (years) } \\
\hline$\leq 50$ & 29 & 23 & \multirow[t]{2}{*}{0.158} \\
\hline$\geq 51$ & 21 & 27 & \\
\hline \multicolumn{4}{|c|}{ Tumor size $(\mathrm{cm})$} \\
\hline$\leq 5$ & 32 & 19 & \multirow[t]{2}{*}{0.008} \\
\hline$>5$ & 18 & 31 & \\
\hline \multicolumn{4}{|c|}{ Tumor grade } \\
\hline I & 22 & 9 & \multirow[t]{3}{*}{0.010} \\
\hline II & 17 & 19 & \\
\hline III & 11 & 22 & \\
\hline \multicolumn{4}{|c|}{ Lymph node metastasis } \\
\hline Yes & 20 & 32 & \multirow[t]{2}{*}{0.014} \\
\hline No & 30 & 18 & \\
\hline \multicolumn{4}{|c|}{ Distant metastasis } \\
\hline Yes & 17 & 28 & \multirow[t]{2}{*}{0.022} \\
\hline No & 33 & 22 & \\
\hline \multicolumn{4}{|c|}{ ER expression } \\
\hline Positive & 27 & 19 & \multirow[t]{2}{*}{0.080} \\
\hline Negative & 23 & 31 & \\
\hline \multicolumn{4}{|c|}{ PR expression } \\
\hline Positive & 29 & 24 & \multirow[t]{2}{*}{0.212} \\
\hline Negative & 21 & 26 & \\
\hline \multicolumn{4}{|c|}{ c-erbB-2 expression } \\
\hline Positive & 22 & 26 & \multirow[t]{2}{*}{0.274} \\
\hline Negative & 28 & 24 & \\
\hline \multicolumn{4}{|c|}{ E-cadherin expression } \\
\hline Positive & 31 & 20 & \multirow[t]{2}{*}{0.022} \\
\hline Negative & 19 & 30 & \\
\hline \multicolumn{4}{|c|}{ Vimentin expression } \\
\hline Positive & 16 & 33 & \multirow[t]{2}{*}{0.013} \\
\hline Negative & 34 & 17 & \\
\hline
\end{tabular}

miR, microRNA; ER, estrogen receptor; progesterone receptor; erbB-2, c-Erb-B2 receptor tyrosine kinase 2.

was negatively associated with RAGE in the fresh tumor tissues (Fig. 1D). RAGE was previously associated with the clinical pathology of breast cancer (8). Analysis of correlation between miR-185-5p and clinical pathological features demonstrated that downregulation of miR-185-5p in breast cancer was related to clinical stage, tumor size $(\mathrm{P}=0.08)$ and grade $(\mathrm{P}=0.010)$, lymphatic metastasis $(\mathrm{P}=0.014)$, and distant metastasis $(\mathrm{P}=0.022)$. However, this downregulation was not associated with age $(\mathrm{P}=0.158)$, ER $(\mathrm{P}=0.080)$, and $\mathrm{PR}(\mathrm{P}=0.212)$ (Table I). These data implied that miR-185-5p expression was significantly related with the clinicopathological features of breast cancer.

miR-185-5p directly targeted RAGE 3'-UTR. miRNAs modulate gene expression via targeting the 3 '-UTR. Bioinformatics analyses of 3'-UTR revealed one putative binding site for
miR-185-5p (Fig. 2A). To study the potential interaction of miR-185-5p and RAGE in breast cancer cells, we predicted that miR-185-5prepressed RAGEexpression via targeting the 3'-UTR. The results suggested that the expression of RAGE protein and mRNA was considerably reduced in MDA231/miR-185-5p cells. By contrast, the expression of RAGE protein and mRNA was markedly upregulated in the MCF-7/anti-miR-185-5p cells than in MCF-7/anti-NC (Fig. 2B, C and D). We cloned the WT or MUT RAGE 3'-UTR of a luciferase reporter gene to investigate whether the predicted targeting site of miR-185-5p on 3'-UTR of RAGE functioned in this regulation. WT or MUT RAGE vector and miR-185-5p or NC were co-transfected into MDA231 cells. The results showed that co-transfection of WT RAGE 3'-UTR and miR-185-5p vector into MDA231 cells notably reduced luciferase activity compared with the co-transfection of control 
A

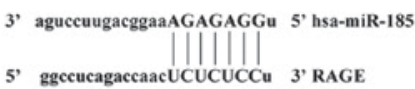

D

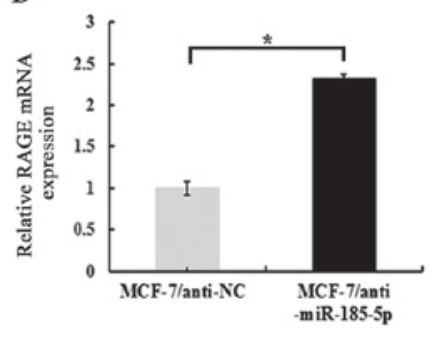

B

$\beta$-actin
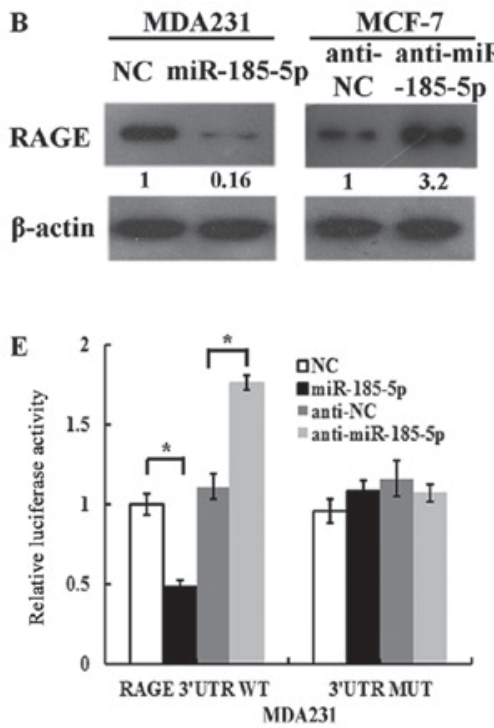
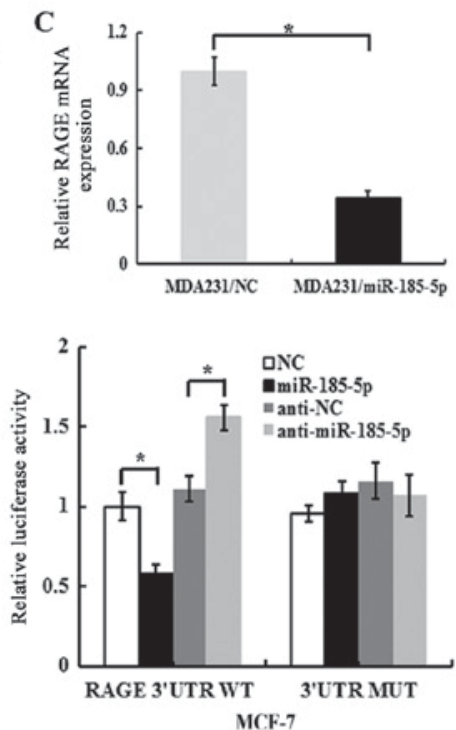

$\mathbf{F}$

MDA231

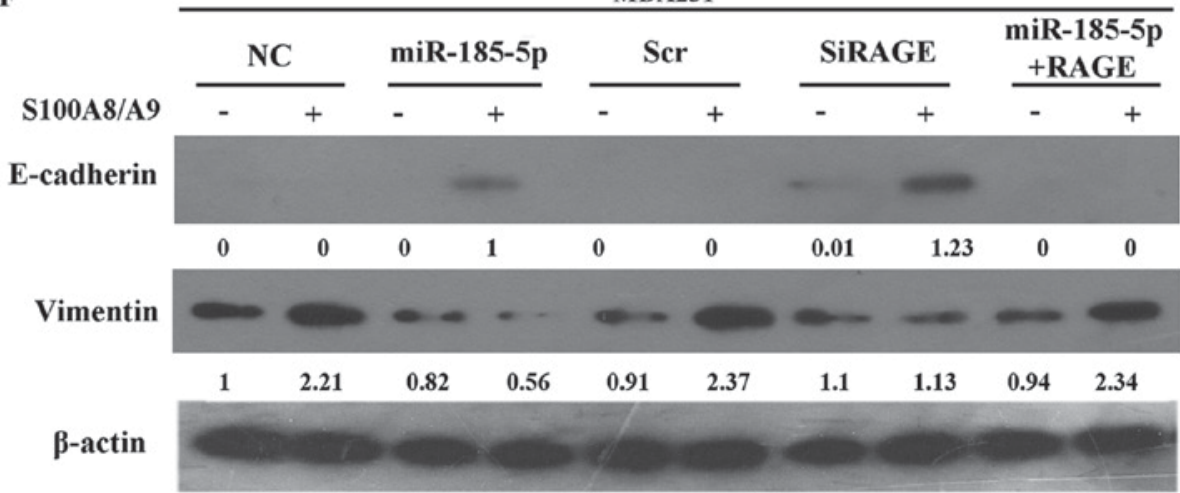

G

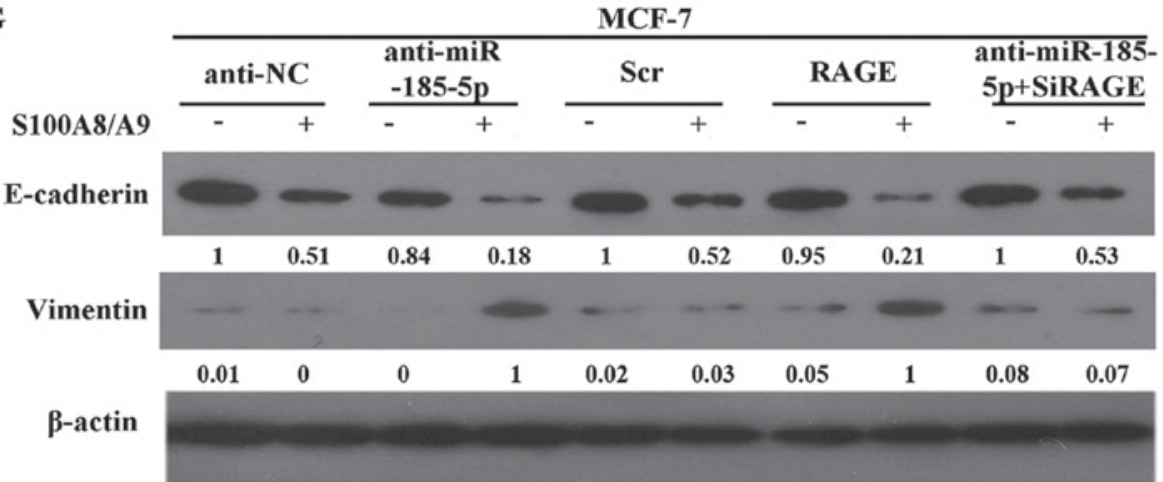

Figure 2. miR-185-5p directly targets RAGE 3'-UTR. (A) Predicted targeting site of miR-185-5p to the 3'-UTRs of RAGE. (B) RAGE protein expression was tested in the indicated cells by western blotting. (C) and (D) Expression levels of RAGE mRNA were detected by reverse transcription-quantitative polymerase chain reaction in the indicated cells. (E) Luciferase activity of pGL3-RAGE 3'-UTR WT or pGL3-RAGE 3'-UTR-MUT report in MDA231 and MCF-7 cells. $(\mathrm{F})$ and $(\mathrm{G})$ Expression of vimentin and E-cadherin was examined in (F) MDA231 and (G) MCF-7. For (B), (F) and (G), the results of western blotting were from a representative of at least three repeated experiments. ${ }^{*} \mathrm{P}<0.05$, as indicated. miR, microRNA; RAGE, advanced glycosylation end-product specific receptor; WT, wild-type; MUT, mutant; NC, negative control; UTR, untranslated region; si-, small interfering RNA; Scr, scramble.

vectors and miR-185-5p (Fig. 2E left). Contrasting results were found in MCF-7 cells co-transfected with anti-miR-185-5p and WT RAGE 3'-UTR (Fig. 2E right). All results showed that miR-185-5p directly targeted 3'-UTR of RAGE and repressed RAGE expression. Subsequently, the regulating relationship between miR-185-5p and RAGE in S100A8/A9-induced EMT was further confirmed. The expression of EMT-related marker was detected after co-transfecting or only transfecting one plasmid. The result evidently showed that the expression of E-cadherin and vimentin was rescued (Fig. 2F). The same results were observed in the MCF-7 cells (Fig. 2G). These findings indicated that miR-185-5p inhibited EMT by modulating RAGE and suppressed invasion.

miR-185-5p inhibited invasion and EMT of breast cancer cells. The expression of miR-185-5p was tested, and the 

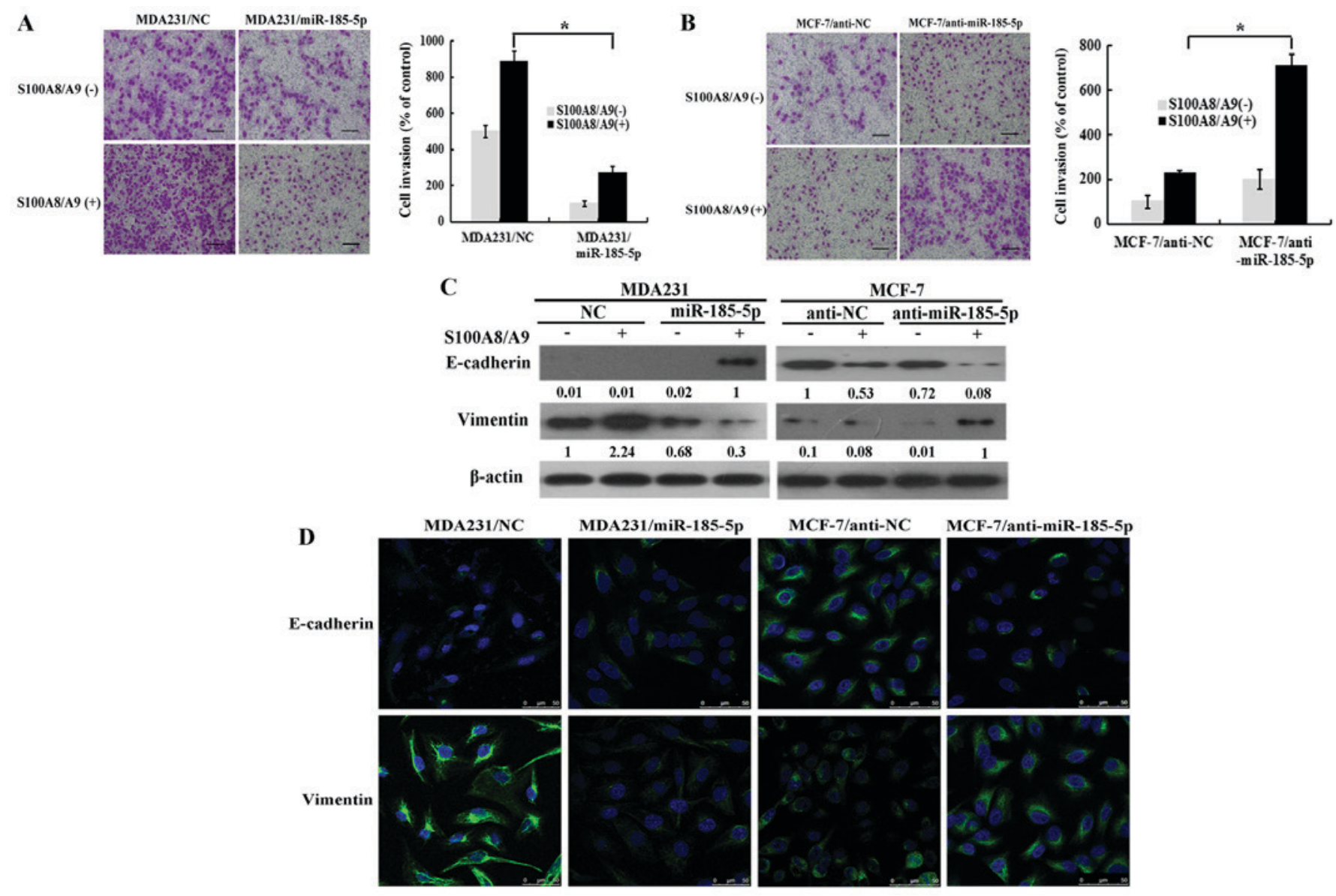

Figure 3. miR-185-5p inhibits the invasion and epithelial mesenchymal transition of breast cancer cells. (A) Invasion capacity of MDA231/miR-185-5p and MDA231/NC cells was analyzed. Left panel, invading cells (magnification, x200); right panel, quantification of penetrating cells. (B) Invasion capacity of MCF-7/anti-miR-185-5p and MCF-7/anti-NC was analyzed. Left panel, invading cells (magnification, x200); right panel, quantification of penetrating cells. (C) The expression of E-cadherin and vimentin was examined in the indicated cells by western blotting. The results of western blotting were from a representative of at least three repeated experiments. (D) Fluorescence microscopy of stained E-cadherin and vimentin in indicated cells (scale bars $50 \mu \mathrm{m}$ ). ${ }^{*} \mathrm{P}<0.05$, as indicated. miR, microRNA; RAGE, advanced glycosylation end-product specific receptor; NC, negative control.

results are shown in Fig. 1A. miR-185-5p was found to be a tumor suppresser gene. The function of miR-185-5p on cell invasion and EMT was further detected. MCF-7 was transfected with anti-NC and anti-miR-185-5p plasmid. Meanwhile, MDA231 was transfected with miR-185-5p and NC plasmid. The invasion ability of MDA231 and MCF-7 was detected by Transwell with or without S100A8/A9 stimulation. The invasion capacity of MDA231/miR-185-5p was notably lower than that of MDA231/NC (Fig. 3A). By contrast, the invasion ability of MCF-7/anti-miR-185-5p was significantly higher than that of MCF-7/anti-NC (Fig. 3B). The results showed that the upregulation of miR-185-5p notably inhibited the invasion. Moreover, the EMT biomarker changed in the breast cancer cell lines after transfection with or without stimulation of S100A8/A9. western blot results showed that vimentin expression was significantly decreased with stimulation of S100A8/A9 in the MDA231/miR-185-5p cells than in MDA231/NC. However, E-cadherin expression in the MDA231/miR-185-5p cells was significantly upregulated (Fig. 3C left). Meanwhile, E-cadherin expression in the MCF-7/anti-miR-185-5p cells was significantly downregulated. The expression of vimentin was significantly upregulated in the MCF-7/anti-miR-185-5p cells than in MCF-7/anti-NC with S100A8/A9 stimulation (Fig. 3C right).
The same results were observed by immunofluorescence staining (Fig. 3D). Hence, miR-185-5p inhibited invasion and EMT.

miR-185-5p inhibited S100A8/A9-induced EMT of breast cancer cells via the $N F-\kappa B /$ Snail signaling pathway. $\mathrm{NF}-\kappa \mathrm{B}$ is a transcription factor cytoplasmic heterodimer protein of the I $\kappa \mathrm{B}$ family and plays an important role in tumor formation. The functional significance of miR-185-5p on cell invasion was elucidated under S100A8/A9 stimulation. The expression of Snail was remarkably downregulated in the MDA231/miR-185-5p cells than in the MDA231/NC cells. However, the expression levels of Twist, Zeb1, and Zeb2 were unchanged in the MDA231/miR-185-5p cells (Fig. 4A). In this study, Snail was downregulated in the nuclei of MDA231/miR-185-5p cells than in the nuclei of MDA231/NC cells under S100A8/A9 stimulation (Fig. 4B). Afterward, $\mathrm{I} \kappa \mathrm{B} \alpha$ phosphorylation was examined to determine whether miR-185-5p mediated the stabilization of Snail through NF- $\kappa$ B activity. The results indicated that $\mathrm{I} \kappa \mathrm{B} \alpha$ phosphorylation was considerably downregulated in MDA231/miR-185-5p cells than in MDA231/NC cells (Fig. 4C). These results proved that miR-185-5p modulated Snail stabilization depending on the activation of $\mathrm{NF}-\kappa \mathrm{B}$. 
$\mathbf{A}$

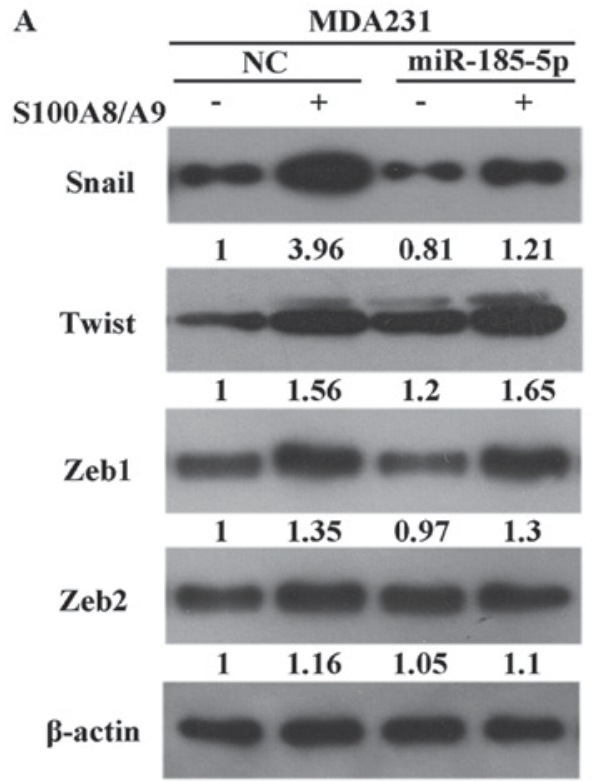

B

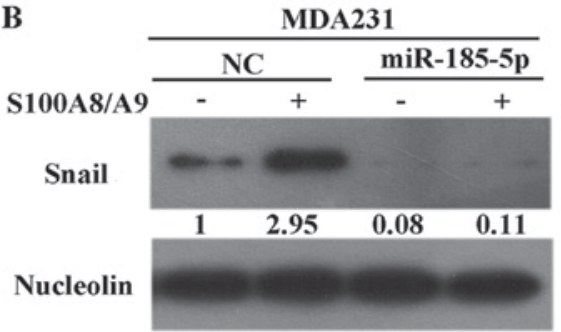

$\mathbf{C}$

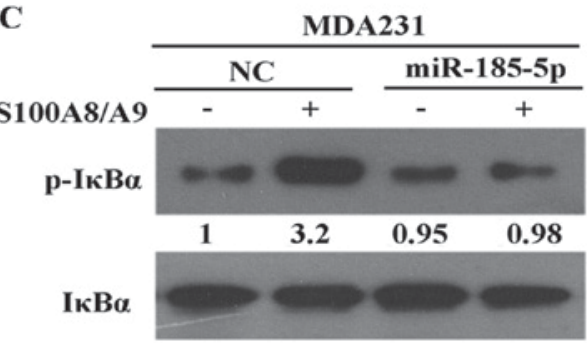

Figure 4. miR-185-5p inhibits S100A8/A9-induced epithelial mesenchymal transition of breast cancer cells via the nuclear factor- $\mathrm{kB} /$ Snail signaling pathway. (A) The expression of Snail, Twist, Zeb1 and Zeb2 was detected in MDA231/miR-185-5p and MDA231/NC cells. (B) Nuclear expression of Snail was examined in the indicated cells. (C) Phosphorylation of IkB $\alpha$ in S100A8/A9-induced MDA231/miR-185-5p and MDA231/NC cells were examined. The results of western blotting were from a representative of at least three repeated experiments. miR, microRNA; NC, negative control; Zeb, zinc finger E-box binding homeobox; Twist, twist-related protein; p-, phosphorylated.

miR-185-5p inhibited S100A8/A9-induced F-actin polymerization of breast cancer cells. The cell chemotaxis assay was designed to detect whether miR-185-5p could inhibit S100A8/A9 and induce chemotaxis of breast cancer cells. No difference in chemotaxis ability was found between MDA231 and MDA231/NC cells. However, this ability was reduced in the MDA231/miR-185-5p cells than in the MDA231/NC cells (Fig. 5A). By contrast, MCF-7/anti-miR-185-5p cells showed an increase in chemotaxis ability (Fig. 5B). The results showed that miR-185-5p acted a vital part in inhibiting chemotaxis of breast cancer cells. Varlet et al (20) found that fine tuning the actin dynamics facilitated cytokinesis. Whether miR-185-5p could mediate the reorganization of F-actin needed further investigation. Thus, we detected F-actin polymerization under miR-185-5p upregulation in breast cancer cells. The quantitative $\mathrm{F}$-actin polymerization experiment indicated that S100A8/A9 induced actin polymerization in MDA231/NC cells at 20 and $60 \mathrm{sec}$, while F-actin polymerization was notably decreased with S100A8/A9 stimulation in the MDA231/miR-185-5p cells (Fig. 5C and D). These results indicated that miR-185-5p strongly limited the cytoskeleton rearrangement and could abolish the formation of stress fibers. Cofilin is an important regulator to balance between F-actin and G-actin. Dephosphorylated cofilin can promote G-actin in forming F-actin, which contributes to cell migration through lamellipodium (21). LIM kinase (LIMK) regulates the structure of the F-actin cytoskeleton by phosphorylation and inactivation of F-actin depolymerization factors of the ADF/cofilin family (22). LIMK is a vital protein in tumor formation and progression $(23,24)$. LIMK or cofilin phosphorylation was proved to be remarkably increased in malignant melanoma (25) and prostate cancer (26). Further analysis indicated that miR-185-5p markedly inhibited the activation of cofilin and LIMK in MDA231/miR-185-5p cells. However, the levels of total LIMK and cofilin remained unchanged (Fig. 5E). These results revealed that miR-185-5p played a vital role in inhibiting F-actin polymerization of breast cancer cells.

Overexpression of miR-185-5p and reduction of $R A G E$ inhibited lung metastasis in SCID mice. To verify the effect of miR-185-5p and RAGE on breast cell invasion in vivo, we injected the axilla of SCID mice with MDA231/NC and MDA231/miR-185-5p cells. Meanwhile, we injected the axilla of SCID mice with SiRAGE/MDA231 and control cells. The number of satellite tumors was counted in sections of SCID mice sacrificed at 10 weeks. The tumor nodules were considerably reduced in MDA231/miR-185-5p cells than in the MDA231/NC group. Upregulation of miR-185-5p resulted in significant inhibition of invasion in vivo. SiRAGE/MDA231 group showed significantly reduced tumor nodules than the control group (Fig. 6A and B). RAGE in sectioned seeded tumors in SCID mice injected with MDA231/NC, Scr/MDA231, SiRAGE/MDA231, and MDA231/miR-185-5p cells were analyzed by western blot. The expression of RAGE was significantly reduced in MDA231/miR-185-5p cells than in MDA231/NC (Fig. 6C). The results consistently indicated that miR-185-5p could inhibit invasion of breast cancer cell in vivo.

\section{Discussion}

An increasing number of studies have recently manifested that the development and progression of malignant tumor is a very complex process and affected by various factors. miRNAs play a vital role in mediating gene by targeting the 3'-UTRs (27), which are vital mediators of cellular functions, such as proliferation (28) and G1/S transition $(29,30)$. Overexpression of miR-185-5p repressed TRIM29 in GC and 
A

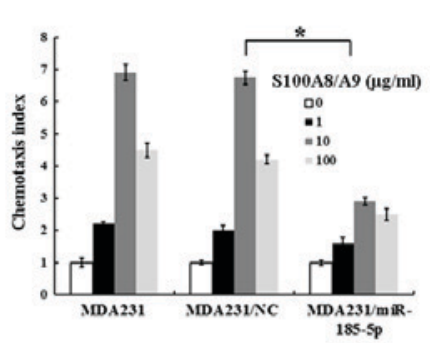

B

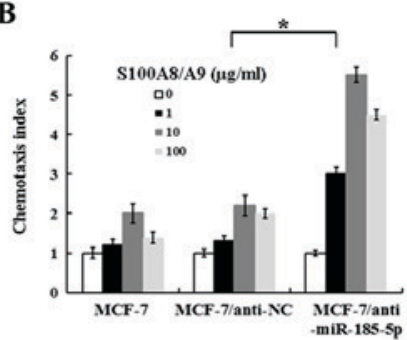

C

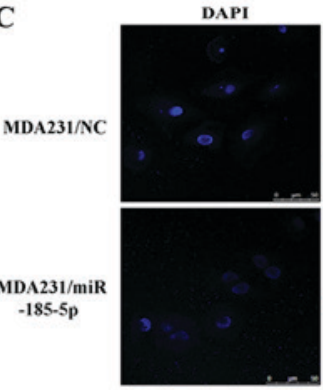

西

E $\operatorname{sio}($
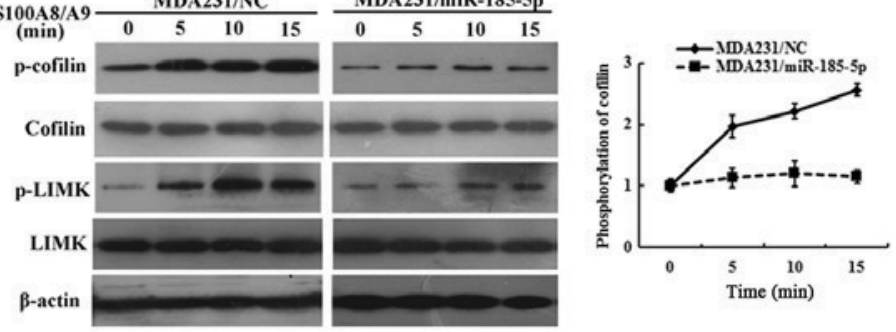
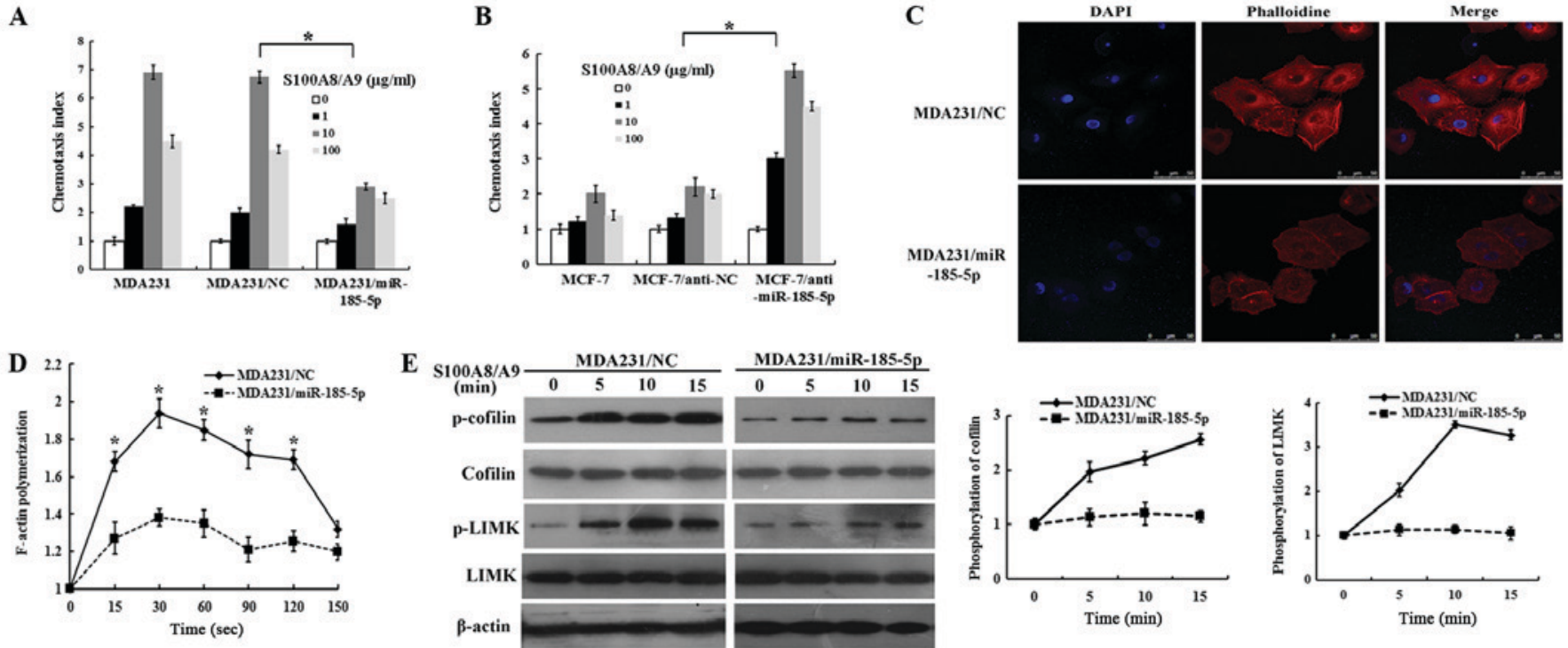

Figure 5. miR-185-5p inhibits S100A8/A9-induced F-actin polymerization of breast cancer cells. Chemotaxis ability was examined with different concentrations of S100A8/A9 stimulation in (A) MDA231, MDA231/miR-185-5p and MDA231/NC cells, and (B) in MCF-7/anti-miR-185-5p, MCF-7/anti-NC and MCF-7 cells. " $\mathrm{P}<0.05$, as indicated (C) F-actin polymerization in indicated cells (scale bars $50 \mu \mathrm{m}$ ). (D) F-actin polymerization in the indicated cells under different periods of stimulation using $10 \mu \mathrm{g} / \mathrm{ml} \mathrm{S100A8/A9.}{ }^{*} \mathrm{P}<0.05 \mathrm{vs}$. MDA231/miR-185-5p. (E) Expression of p-cofilin and p-LIMK in MDA231 cells under $10 \mu \mathrm{g} / \mathrm{ml} \mathrm{S100A8/A9} \mathrm{stimulation.} \mathrm{The} \mathrm{results} \mathrm{of} \mathrm{western} \mathrm{blotting} \mathrm{were} \mathrm{from} \mathrm{a} \mathrm{representative} \mathrm{of} \mathrm{at} \mathrm{least} \mathrm{three} \mathrm{repeated} \mathrm{experiments.} \mathrm{miR,} \mathrm{microRNA;} \mathrm{NC,}$ negative control; LIMK, LIM kinase; p-, phosphorylated.

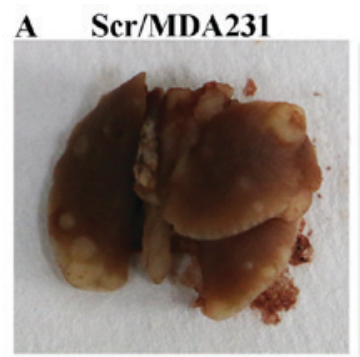

SiRAGE/MDA231
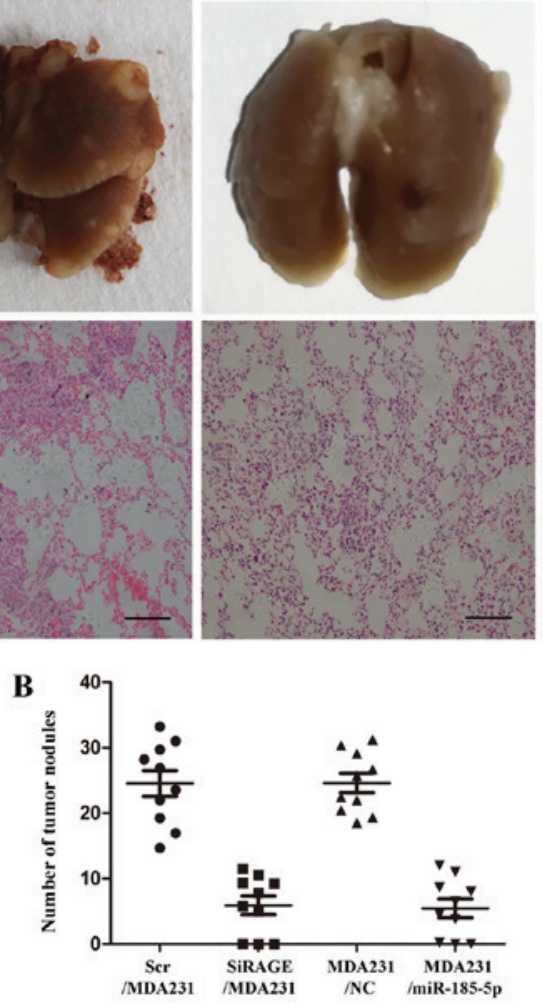

MDA231/NC
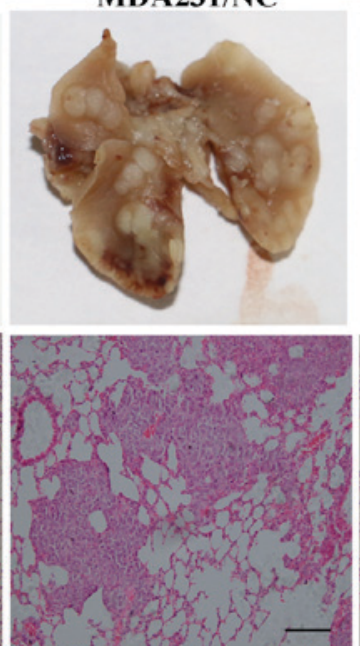

C

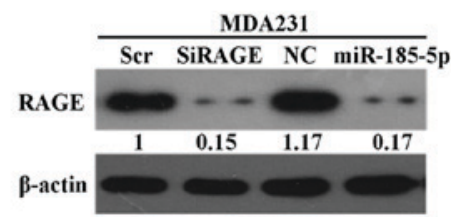

Figure 6. Overexpression of miR-185-5p and reduction of RAGE inhibits lung metastasis in SCID mice. (A) Lung metastasis and hematoxylin and eosin staining of the respective tissues from SCID mice. Scale bars, $50 \mu \mathrm{m}$. (B) The number of lung metastasis nodes were calculated (n=10). (C) RAGE expression was detected in SCID xenograft tumors. The results of western blotting were from a representative of at least three repeated experiments. miR, microRNA; NC, negative control; SCID, Severe Combined Immune-deficiency; RAGE, advanced glycosylation end-product specific receptor; Scr, scramble.

significantly inhibited malignant behavior (31). Meanwhile, miR-185 repressed hepatocellular carcinoma tumor genesis by targeting the DNMT1/PTEN/Akt signal pathway (32).
miR-185-5p has been demonstrated to directly target RAGE and inhibit the invasion and metastasis in ESCC. However, the important molecular mechanism by which miR-185-5p targets 
RAGE to repress breast cancer remains unknown. In this study, the data revealed that miR-185-5p directly targeted and modulated RAGE 3'-UTR and inhibited invasion and EMT. In addition, this miRNA sufficiently reversed the mesenchymal potentials and reduced the invasiveness of mesenchymal cells.

EMT is an important molecular mechanism that determines the metastasis and invasion of carcinoma cells (33). Consistent with previous finding, we previously showed that the combination of RAGE-S100A8/A9 promoted the invasion and metastasis of breast cancer cells (8). Meanwhile, EMT regulated multiple signaling pathways, such as TGF- $\beta$ (34), PI3K, MAPK, and $\mathrm{Wnt} / \beta$-catenin. Numerous genes and transcription factors, such as Snail, Twist, and ZEB1/2 $(35,36)$, have been found as key regulators in EMT. Zhai et al (37) indicated that miR-143 inhibited migration and invasion by downregulating ERK5 in breast cancer cells. In this study, miR-185-5p inhibited the S100A8/A9-induced EMT via NF-кB/Snail signaling pathway.

Among the multifaceted effects exerted by this program, rearrangement of actin cytoskeleton is the major driving mechanism for cell migration and invasion in the development of EMT and cancer cells with invasive behavior (38). Wang et al (39) found that MIIP could inhibit endometrial carcinoma migration via cytoskeleton reorganization with markedly reduced formation of lamellipodia. Previous studies indicated that HSPB8 regulated BAG3 levels through a mechanism that involves effects on branched actin nucleation in cancer cells (20). Phosphorylated cofilin could process the polymerization of F-actin and formation of lamellipodium, leading to the damage of lamellipodia-based metastasis. Hence, these results indicated that miR-185-5p inhibited F-actin polymerization. miR-185-5p has a significant part in metastasis and invasion of breast cancer cells.

S100 family members have diverse functions in cancer development. S100A8/A9, which is a member of the S100 protein family, contributes to the progression of human cancers. Previous studies showed that modulating the MMP-2 expression S100A8/A9 related to carcinoma cell phenotype and activity can regulate carcinoma cell metastasis $(40,41)$. S100A8/A9 is released at the region of inflammation by phagocytes, monocytes, epithelial cells, and endothelial cells (42). This protein regulates various processes during chronic inflammation. The results show that the S100A8/A9 protein dimers interact with RAGE at cytomembrane of melanoma tumor cells $(43,44)$. We previously reported the impact of the RAGE-S100A8/A9 interaction on cell invasion and metastasis formation in breast cancer $(8,45)$. Here, we demonstrated the miR-185-5p targeting of RAGE in metastasis and invasive capabilities via combining to extracellular S100A8/A9. Hence, miR-185-5p inhibited the invasion and migration of breast cancer cells by targeting RAGE.

Thus, a relation between miR-185-5p and RAGE expression was established in this study. The importance of miR-185-5p regulation of $\mathrm{F}$-actin polymerization and reversal of EMT in breast cancer was determined. The NF- $\mathrm{B} / \mathrm{Snail}$ signaling pathway was involved in the suppression of EMT. Furthermore, all findings demonstrated that miR-185-5p played a role in inhibiting invasion and migration in vivo. These results provided a new rationale for using miR-185-5p to target RAGE, which is a novel strategy for breast cancer therapy.

\section{Acknowledgements}

Not applicable.

\section{Funding}

The present study was supported by the National Natural Science Foundation of China (grant nos. 81702932, 81641111 and 81402389) and the Natural Science Foundation of Shandong Province (grant no. ZR2015HL065).

\section{Availability of data and materials}

The datasets used and analyzed during the current study are available from the corresponding author on reasonable request.

\section{Authors' contributions}

CY and GZ performed the experiments and wrote the manuscript. RS, XP and XW analyzed data. HL and YS assisted with the design of the experiments. All authors read and approved the final manuscript.

\section{Ethics approval and consent to participate}

Written informed consent was acquired from all patients, and the study was approved by the Institute Research Ethics Committee at the Cancer Center, Weifang Medical University.

\section{Patient consent for publication}

Written informed consent was acquired from all patients.

\section{Competing interests}

The authors declare that they have no competing interests.

\section{References}

1. DeSantis C, Ma J, Bryan L and Jemal A: Breast cancer statistics, 2013. CA Cancer J Clin 64: 52-62, 2014.

2. Malik P, Chaudhry N, Mittal R and Mukherjee TK: Role of receptor for advanced glycation end products in the complication and progression of various types of cancers. Biochim Biophys Acta 1850: 1898-1904, 2015.

3. Jules J, Maiguel D and Hudson BI: Alternative splicing of the RAGE cytoplasmic domain regulates cell signaling and function. PLoS One 8: e78267, 2013.

4. Chen X, Zhang L, Zhang IY, Liang J, Wang H, Ouyang M, Wu S, da Fonseca ACC, Weng L, Yamamoto Y, et al: RAGE expression in tumor-associated macrophages promotes angiogenesis in glioma. Cancer Res 74: 7285-7297, 2014.

5. Elangovan I, Thirugnanam S, Chen A, Zheng G, Bosland MC, Kajdacsy-Balla A and Gnanasekar M: Targeting receptor for advanced glycation end products (RAGE) expression induces apoptosis and inhibits prostate tumor growth. Biochem Biophys Res Commun 417: 1133-1138, 2012.

6. Kalea AZ, See F, Harja E, Arriero M, Schmidt AM and Hudson BI: Alternatively spliced RAGEv1 inhibits tumorigenesis through suppression of JNK signaling. Cancer Res 70: 5628-5638, 2010

7. Kang R, Tang D, Schapiro NE, Livesey KM, Farkas A, Loughran P, Bierhaus A, Lotze MT and Zeh HJ: The receptor for advanced glycation end products (RAGE) sustains autophagy and limits apoptosis, promoting pancreatic tumor cell survival. Cell Death Differ 17: 666-676, 2010. 
8. Yin C, Li H, Zhang B, Liu Y, Lu G, Lu S, Sun L, Qi Y, Li X and Chen W: RAGE-binding S100A8/A9 promotes the migration and invasion of human breast cancer cells through actin polymerization and epithelial-mesenchymal transition. Breast Cancer Res Treat 142: 297-309, 2013.

9. Gonzalez DM and Medici D: Signaling mechanisms of the epithelial-mesenchymal transition. Sci Signal 7: re8, 2014.

10. Lamouille S, Xu J and Derynck R: Molecular mechanisms of epithelial-mesenchymal transition. Nat Rev Mol Cell Biol 15: 178-196, 2014.

11. Tseng JH, Bisogna M, Hoang LN, Olvera N, Rodriguez-Aguayo C, Lopez-Berestein G, Sood AK, Levine DA and Jelinic P: miR-200c-driven Mesenchymal-To-Epithelial Transition is a Therapeutic Target in Uterine Carcinosarcomas. Sci Rep 7: 3614, 2017.

12. Siemens H, Jackstadt R, Hünten S, Kaller M, Menssen A, Götz U and Hermeking H: miR-34 and SNAIL form a double-negative feedback loop to regulate epithelial-mesenchymal transitions. Cell Cycle 10: 4256-4271, 2011.

13. Gwak JM, Kim HJ, Kim EJ, Chung YR, Yun S, Seo AN, Lee HJ and Park SY: MicroRNA-9 is associated with epithelial-mesenchymal transition, breast cancer stem cell phenotype, and tumor progression in breast cancer. Breast Cancer Res Treat 147: 39-49, 2014.

14. Li S, Ma Y, Hou X, Liu Y, Li K, Xu S and Wang J: MiR-185 acts as a tumor suppressor by targeting AKT1 in non-small cell lung cancer cells. Int J Clin Exp Pathol 8: 11854-11862, 2015.

15. Yoon JH, Choi YJ, Choi WS, Ashktorab H, Smoot DT, Nam SW, Lee JY and Park WS: GKN1-miR-185-DNMT1 axis suppresses gastric carcinogenesis through regulation of epigenetic alteration and cell cycle. Clin Cancer Res 19: 4599-4610, 2013.

16. Wang R, Tian S, Wang HB, Chu DP, Cao JL, Xia HF and Ma X: MiR-185 is involved in human breast carcinogenesis by targeting Vegfa. FEBS Lett 588: 4438-4447, 2014.

17. Tang $\mathrm{H}$, Wang $\mathrm{Z}$, Liu X, Liu $\mathrm{Q}, \mathrm{Xu} \mathrm{G}, \mathrm{Li} \mathrm{G}$ and $\mathrm{Wu} \mathrm{M}$ : LRRC4 inhibits glioma cell growth and invasion through a miR-185-dependent pathway. Curr Cancer Drug Targets 12: 1032-1042, 2012.

18. Jing R, Chen W, Wang H, Ju S, Cong H, Sun B, Jin Q, Chu S, $\mathrm{Xu} \mathrm{L}$ and Cui M: Plasma miR-185 is decreased in patients with esophageal squamous cell carcinoma and might suppress tumor migration and invasion by targeting RAGE. Am J Physiol Gastrointest Liver Physiol 309: G719-G729, 2015.

19. Livak KJ and Schmittgen TD: Analysis of relative gene expression data using real-time quantitative PCR and the 2(-Delta Delta C(T)) method. Methods 25: 402-408, 2001.

20. Varlet AA, Fuchs M, Luthold C, Lambert H, Landry J and Lavoie JN: Fine-tuning of actin dynamics by the HSPB8-BAG3 chaperone complex facilitates cytokinesis and contributes to its impact on cell division. Cell Stress Chaperones 22: 553-567, 2017

21. Shi C, Cai Y, Li Y, Li Y, Hu N, Ma S, Hu S, Zhu P, Wang W and Zhou H: Yap promotes hepatocellular carcinoma metastasis and mobilization via governing cofilin/F-actin/lamellipodium axis by regulation of JNK/Bnip3/SERCA/CaMKII pathways. Redox Biol 14: 59-71, 2018.

22. Fife CM, McCarroll JA and Kavallaris M: Movers and shakers: Cell cytoskeleton in cancer metastasis. Br J Pharmacol 171: 5507-5523, 2014

23. Manetti F: LIM kinases are attractive targets with many macromolecular partners and only a few small molecule regulators. Med Res Rev 32: 968-998, 2012.

24. Mali RS and Kapur R: Targeting Rho associated kinases in leukemia and myeloproliferative neoplasms. Oncotarget 3 : 909-910, 2012

25. Okamoto I, Pirker C, Bilban M, Berger W, Losert D, Marosi C, Haas OA, Wolff K and Pehamberger H: Seven novel and stable translocations associated with oncogenic gene expression in malignant melanoma. Neoplasia 7: 303-311, 2005.

26. Mardilovich K, Gabrielsen M, McGarry L, Orange C, Patel R, Shanks E, Edwards J and Olson MF: Elevated LIM kinase in nonmetastatic prostate cancer reflects its role in facilitating androgen receptor nuclear translocation. Mol Cancer Ther 14: 246-258, 2015.

27. Zhi Q, Zhu J, Guo X, He S, Xue X, Zhou J, Hu B, Li H, Chen S, Zhao H and Kuang Y: Metastasis-related miR-185 is a potential prognostic biomarker for hepatocellular carcinoma in early stage. Biomed Pharmacother 67: 393-398, 2013.
28. Osbourne A, Calway T, Broman M, McSharry S, Earley J and Kim GH: Downregulation of connexin43 by microRNA-130a in cardiomyocytes results in cardiac arrhythmias. J Mol Cell Cardiol 74: 53-63, 2014

29. Liao X, Xue H, Wang YC, Nazor KL, Guo S, Trivedi N, Peterson SE, Liu Y, Loring JF and Laurent LC: Matched miRNA and mRNA signatures from an hESC-based in vitro model of pancreatic differentiation reveal novel regulatory interactions. J Cell Sci 126: 3848-3861, 2013.

30. James EN, Delany AM and Nair LS: Post-transcriptional regulation in osteoblasts using localized delivery of miR-29a inhibitor from nanofibers to enhance extracellular matrix deposition. Acta Biomater 10: 3571-3580, 2014.

31. Qiu F, Xiong JP, Deng J and Xiang XJ: TRIM29 functions as an oncogene in gastric cancer and is regulated by miR-185. Int $\mathrm{J}$ Clin Exp Pathol 8: 5053-5061, 2015.

32. Qadir XV, Han C, Lu D, Zhang J and Wu T: miR-185 inhibits hepatocellular carcinoma growth by targeting the DNMT1/PTEN/Akt pathway. Am J Pathol 184: 2355-2364, 2014.

33. Ponnusamy MP, Seshacharyulu P, Lakshmanan I, Vaz AP, Chugh S and Batra SK: Emerging role of mucins in epithelial to mesenchymal transition. Curr Cancer Drug Targets 13: 945-956, 2013.

34. Wu Y, Tran T, Dwabe S, Sarkissyan M, Kim J, Nava M, Clayton S, Pietras R, Farias-Eisner R and Vadgama JV: A83-01 inhibits TGF- $\beta$-induced upregulation of Wnt3 and epithelial to mesenchymal transition in HER2-overexpressing breast cancer cells. Breast Cancer Res Treat 163: 449-460, 2017.

35. Batlle E, Sancho E, Francí C, Domínguez D, Monfar M, Baulida J and García De Herreros A: The transcription factor snail is a repressor of E-cadherin gene expression in epithelial tumour cells. Nat Cell Biol 2: 84-89, 2000.

36. Yang J, Mani SA, Donaher JL, Ramaswamy S, Itzykson RA, Come C, Savagner P, Gitelman I, Richardson A and Weinberg RA: Twist, a master regulator of morphogenesis, plays an essential role in tumor metastasis. Cell 117: 927-939, 2004.

37. Zhai L, Ma C, Li W, Yang S and Liu Z: miR-143 suppresses epithelial-mesenchymal transition and inhibits tumor growth of breast cancer through down-regulation of ERK5. Mol Carcinog 55: 1990-2000, 2016

38. Yilmaz M and Christofori G: EMT, the cytoskeleton, and cancer cell invasion. Cancer Metastasis Rev 28: 15-33, 2009.

39. Wang Y, Hu L, Ji P, Teng F, Tian W, Liu Y, Cogdell D, Liu J, Sood AK, Broaddus R, et al: Erratum to: MIIP remodels Rac1-mediated cytoskeleton structure in suppression of endometrial cancer metastasis. J Hematol Oncol 10: 163, 2017.

40. Silva EJ, Argyris PP, Zou X, Ross KF and Herzberg MC: S100A8/A9 regulates MMP-2 expression and invasion and migration by carcinoma cells. Int $\mathrm{J}$ Biochem Cell Biol 55: 279-287, 2014.

41. Khammanivong A, Wang C, Sorenson BS, Ross KF and Herzberg MC: S100A8/A9 (calprotectin) negatively regulates G2/M cell cycle progression and growth of squamous cell carcinoma. PLoS One 8: e69395, 2013.

42. Gebhardt C, Németh J, Angel P and Hess J: S100A8 and S100A9 in inflammation and cancer. Biochem Pharmacol 72: 1622-1631, 2006.

43. Ichikawa M, Williams R, Wang L, Vogl T and Srikrishna G: S100A8/A9 activate key genes and pathways in colon tumor progression. Mol Cancer Res 9: 133-148, 2011.

44. Saha A, Lee YC, Zhang Z, Chandra G, Su SB and Mukherjee AB: Lack of an endogenous anti-inflammatory protein in mice enhances colonization of B16F10 melanoma cells in the lungs. J Biol Chem 285: 10822-10831, 2010.

45. Dahlmann M, Okhrimenko A, Marcinkowski P, Osterland M, Herrmann P, Smith J, Heizmann CW, Schlag PM and Stein U: RAGE mediates S100A4-induced cell motility via MAPK/ERK and hypoxia signaling and is a prognostic biomarker for human colorectal cancer metastasis. Oncotarget 5: 3220-3233, 2014.

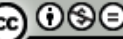

This work is licensed under a Creative Commons Attribution-NonCommercial-NoDerivatives 4.0 International (CC BY-NC-ND 4.0) License. 